\title{
Viewpoint
}

\section{Via satellite}

\section{David Rubincam}

Planetary Geodynamics Laboratory NASA Goddard Space Flight Center, Greenbelt, MD 20771, USA

Published November 29, 2010

More than a decade's worth of data collected from the LAGEOS II satellite is offering a new way to test general relativity.

Subject Areas: Gravitation

\author{
A Viewpoint on: \\ Accurate Measurement in the Field of the Earth of the General-Relativistic Precession of the LAGEOS II Pericen- \\ ter and New Constraints on Non-Newtonian Gravity \\ David M. Lucchesi and Roberto Peron \\ Phys. Rev. Lett. 105, 231103 (2010) - Published November 29, 2010
}

Testing Einstein's general theory of relativity against the trajectories of astronomical bodies has enjoyed a long history. The very first test was the perihelion advance of Mercury: once the gravitational effects of the other planets were removed, the innermost planet's eccentric orbit showed a residual advance that did not follow Newton's formulation of gravity.

Now, nearly a century later, David Lucchesi and Roberto Peron at the Istituto Nazionale di Astrofisica in Rome are again testing general relativity [1] using the pericenter advance of an astronomical body-only this time, it is one made by man (see Fig. 1). In their paper, which appears in Physical Review Letters, they show that once again, Einstein has passed with flying colors.

Mercury's anomalous perihelion advance of 43 arcseconds per century was already well known when Albert Einstein - a savant if ever there was one-produced his theory. General relativity had to get that number; there were no adjustable parameters to absorb it. When he found his theory nailed it within the error bars of the time, Einstein was ecstatic for days, and even had palpitations of the heart [2]. In the years thereafter, there have been many astronomical tests of general relativity, including the deflection of starlight near the Sun [3], gravitational lensing by galaxies (e.g., Ref. [4]), the decay of the orbits of binary pulsars that results from gravitational radiation [5], and the path of the Moon's orbit [6].

The ability to laser-track the Moon's orbit came in the 1960s and 1970s, when the Apollo astronauts installed retroreflector arrays on the Moon. Other arrays were deposited on the lunar surface by soft-landing unmanned Russian spacecraft. Laser beams sent from Earth and reflected off the arrays are still returning a wealth of scientific information, including a measurement of the $4 \mathrm{~cm}$ per year recession of the Moon that results from the tides

DOI: $10.1103 /$ Physics.3.100

URL: http://link.aps.org/doi/10.1103/Physics.3.100

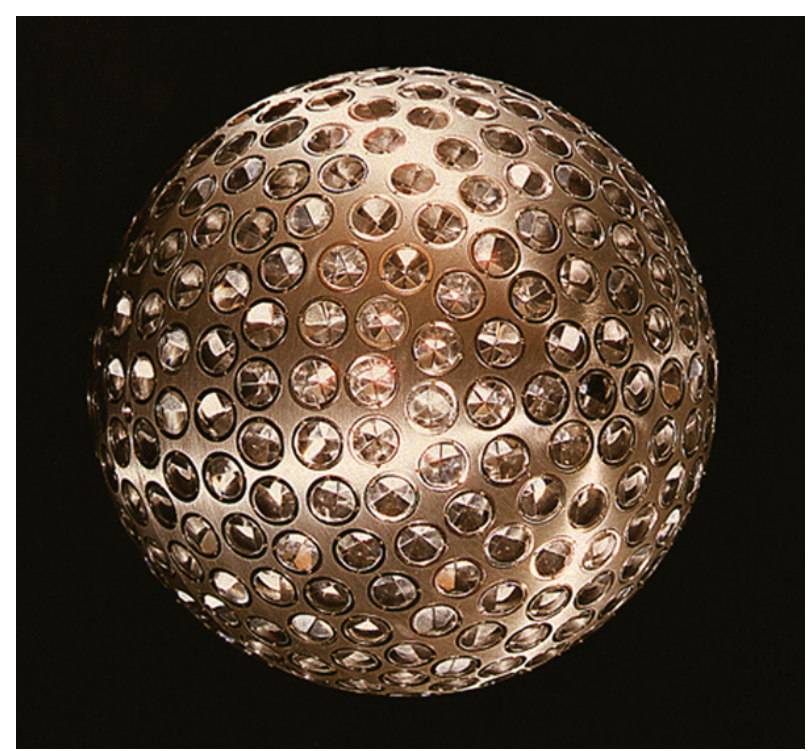

FIG. 1: Photograph of the LAGEOS II satellite. (Credit: NASA)

on the Earth pushing the Moon away [7].

Even though Russia launched the first artificial satellite to orbit the Earth in 1957 (more than a decade before the first Apollo landing in 1969), satellites are a Johnnycome-lately to tests of general relativity compared to natural bodies like Mercury, pulsars, and even our nearest neighbor, the Moon. The reasons for this tardiness are not hard to find. The near-Earth environment sampled by satellites is much busier than the cleaner realm presided over by the Moon. Moreover, because they have a higher surface-area-to-mass ratio, satellites are deflected by forces scarcely noticed by our ponderous neighbor. The many factors affecting the motion of a satellite therefore had to be sorted out before the small 
relativistic effects could become visible.

One factor is the Earth's gravitational field, which is multipole, due to lumps and bumps inside the Earth, rather than monopole. These multipole terms largely die out at the Moon's distance of 60 Earth radii, but are significant for satellites orbiting near the Earth. In particular, besides relativity, our planet's oblateness, which is mainly a result of the Earth's rotation, causes the long axis of a satellite's elliptical orbit to precess. This and other terms in the multipole expansion of the Earth's gravitational field must be found in order to disentangle the relativistic advance of the perigee from the mundane gravity anomalies, but obtaining an accurate picture of the Earth's gravitational field has taken years of analyzing many satellites, in many different orbits (e.g., Ref. [8]).

The complexities of the near-Earth environment don't end with its multipole field. Tides sloshing about on the Earth affect a satellite's motion: when water moves, it takes its gravitational pull with it. The pull from the Sun, Moon, and other planets must be reckoned with as well. But at least gravitational forces obeying the equivalence principle accelerate satellites independent of their mass, and virtually independently of their size and shape; in other words, a satellite may be considered a point-mass. This is not true of the nongravitational forces, often called "surface forces" in space geodesy.

Atmospheric drag and solar radiation pressure are the principal surface forces. The atmosphere is so thin and the area-to-mass ratio so favorable to the Moon that drag scarcely affects it. For most Earth satellites, however, being close to the Earth, where the atmosphere is denser, and having poor area-to-mass ratios, drag affects them so badly that they eventually make a fiery reentry and burn up in a blaze of glory in the atmosphere. Solar radiation pressure also depends on area-to-mass. Though the momentum transfers from solar photons to a satellite are small, woe betide any orbit computing program that ignores them. Photon thrust from asymmetric thermal forces, first discovered by Ivan Yarkovsky, must also be given its due.

All of the above factors, and several more, must be sorted out and included in the massive computer programs that integrate over the path of satellite orbits before their various parameters can be estimated [9]. Much of the data for these programs come from satellite laser ranging (SLR) - a worldwide network of laser stations that send signals to retroreflector-carrying satellites. The time that it takes a laser pulse to go up and back gives the range, and eventually, the orbit. These retroreflectors are much like those in the arrays left on the Moon: fused silica corner cubes that utilize total internal reflection. Taking all the data, developing the computer programs, and recovering the parameters associated with the many factors that affect the satellite orbits has been a heroic and largely unsung effort, requiring many years of work.

The focus of this decades-long effort was, and still is, mainly to understand the geophysics of the Earth and the space physics of the near-Earth environment. However, once these became well enough known, researchers were finally in a good position to use satellites to study the effects of general relativity. And although they were late to the party when it came to testing Einstein, satellites are now beginning to pay off handsomely. A major triumph was Ignazio Ciufolini and Erricos Pavlis's measurement in 2004 of the LenseThirring effect, which is caused by the Earth's rotation dragging the reference frame. By looking at the motion of the nodes of the LAGEOS I and LAGEOS II satellites [10], Ciufolini and Pavlis measured the Lense-Thirring effect with an error of $10 \%$ (the node is an intersection of a satellite's orbit plane with the equatorial plane). Gravity Probe B, a satellite mission that measures framedragging with gyroscopes, currently reports agreement with Einstein at the $14 \%$ level [11].

LAGEOS I and its nearly identical twin LAGEOS II are dense satellites designed to minimize the surface forces. Each has a spherical shape to make its response to these forces as independent of orientation as possible. They are at high enough altitude so that atmospheric drag is not a major problem (drag will not bring them down to Earth for millions of years). Both satellites are completely passive, in that they contain no electronics whatever. Instead, they serve as high-tech targets for the SLR network. Their shiny aluminum exteriors are studded with retroreflectors (see Fig. 1), so that the reflection of laser pulses to Earth is guaranteed, regardless of orientation.

Lucchesi and Peron concentrated on one of these satellites, LAGEOS II, for their study-the rationale being that LAGEOS II has the more eccentric orbit, so that the swinging around of the perigee is easier to observe. The GEODYN program, developed under the leadership of David E. Smith at NASA, and the UTOPIA program, developed under the leadership of Byron Tapley at the University of Texas, are two programs commonly used to compute satellite orbits. For their study, Lucchesi and Peron chose GEODYN II, a later version of GEODYN.

Using thirteen years' worth of data, Lucchesi and Peron recovered the total secular (long term) perigee shift due to three relativistic effects: the Einstein, deSitter, and Lense-Thirring precessions. Of these, the LenseThirring effect accounts for $1.7 \%$ of the predicted total. In the total for all three effects, there was a fractional discrepancy of only $2.8 \times 10^{-4}$ in the trend of the observed precession rate and that predicted by general relativity.

Lucchesi and Peron also consider whether gravity obeys a Yukawa potential rather than Einstein's theory. On the scale of the distance between the Earth and the satellite $(\sim 6000 \mathrm{~km})$, they once again find good agreement with Einstein: any Yukawa-type interaction must be extremely weak, with a result consistent with zero. So it appears that general relativity has once more passed another set of tests, a remarkable achievement for a the- 
ory that is now nearly a century old, and almost a third the age of Newton's Principia Mathematica.

\section{References}

[1] D. M. Lucchesi and R. Peron, Phys. Rev. Lett. 105, 231103 (2010).

[2] A. Pais, Subtle Is the Lord: the Science and Life of Albert Einstein (Oxford University Press, New York, 1982).
[3] F. W. Dyson et al., Philos. Trans. R. Soc. London 220, 291 (1920).

[4] T. Treu, Annu. Rev. Astron. Astrophys. 48, 87 (2010).

[5] J. H. Taylor and J. M. Weisberg, Astrophys. J. 253,908, 1982.

[6] J. G. Williams et al., Phys. Rev. Lett. 93, 261101 (2004).

[7] J. O. Dickey et al., Science 265, 482 (1994).

[8] F. J. Lerch et al., J. Geophys. Res. 99, 2815 (1994).

[9] D. E. Smithet al., LAGEOS Geodetic Analysis-SL7.1, Report No. NASA-TM-104549, September 1991.

[10] I. Ciufolini and E. C. Pavlis, Nature 431, 958 (2004).

[11] http://einstein.stanford.edu/.

\section{About the Author}

\section{David Rubincam}

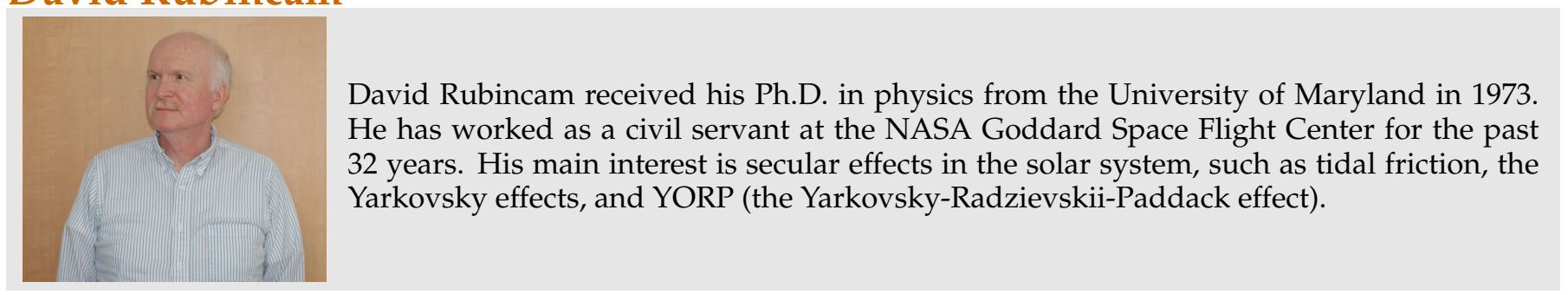

